\title{
Aerosol generation from high speed ophthalmic instrumentation and the risk of contamination from SARS COVID19
}

\author{
Zachariah R. Koshy ${ }^{1} \cdot$ David Dickie $^{2}$
}

Received: 13 May 2020 / Revised: 18 May 2020 / Accepted: 20 May 2020 / Published online: 4 June 2020

(c) The Royal College of Ophthalmologists 2020

The current COVID 19 pandemic has raised questions about the aerosol generating potential of ophthalmic procedures, primarily that of phacoemulsification and vitrectomy. Both of these procedures involve high speed instrumentation, a characteristic associated with aerosol generation in other fields [1]. The transmission of virus particles through infected aerosol and droplet is the primary mode of contagion in SARS COVID19. The above factors have prompted the RCOphth to deem both these procedures as potentially aerosol generating along with other national professional bodies in ophthalmology.

This necessitates a higher degree of personal protective equipment (PPE) and protocols for the team carrying out these procedures, than would be the case if the risk was from droplet transmission alone. This includes the PPE worn by staff, duration of procedure time in theatre, decontamination of equipment and surfaces in theatre and sequencing of various steps including donning and doffing of PPE. Full PPE for aerosol generating procedures (AGP) includes face shields or protective goggles and can limit the performance of the surgeon. This has implications on cost, efficiency and effectiveness of delivery of these procedures, which form a significant part of ophthalmic care.

The main source of infected droplets and aerosol is well documented as the naso-pharyngeal mucosa. Recent evidence suggests a low risk of transmission from the tear film itself [2].

There is no reliable, current evidence to support or discount phacoemulsification and vitrectomy as AGPs. Phacoemulsification technology uses an ultrasonic hand piece

Zachariah R. Koshy

zkoshy@doctors.org.uk

1 Department of Ophthalmology, University Hospitals Ayr and Crosshouse, Ayr KA6 6DX, UK

2 Dept of Medical Illustration, University Hospital Ayr and Crosshouse, Scotland, UK which has a metallic needle tip that vibrates along a longitudinal axis typically around $40,000 \mathrm{~Hz}$ [3]. A torsional phaco tip oscillates around the longitudinal axis at 32,000 $\mathrm{Hz}$ with bursts of longitudinal vibration [4]. The shaft of the needle is covered by a polymer sleeve that also allows a flow of fluid which acts to diffuse heat from the needle as well as cushioning any vibrations external to the tip. The assumption is that the vibrating tip of the needle can potentially generate aerosol from the fluid layer on the ocular surface, which is a mixture of tears and the copious amount of fluid from the infusion stream (which would have a dilution effect too).

A conventional vitrectomy hand piece incorporates a blade within a tubular structure, which oscillates along a longitudinal axis to deliver anywhere up to 8000 cuts per minute (cpm or $130 \mathrm{~Hz})$. The mechanical vibration is assumed to be the cause of the aerosol generation.

There is a spectrum of particle size and associated behaviour for sources that generate droplets and aerosols from high speed devices. Larger droplets follow a parabolic trajectory and settle in the immediate vicinity of the source. Aerosols comprised of particles typically $<50 \mu \mathrm{m}$, have a floating property and can disperse widely [5].

We attempted to demonstrate droplet/aerosol generation with the above instruments both in vivo and in vitro using different settings. We aimed to capture visible aerosol generation using high definition, slow motion videography (Nikon Z6, $36 \mathrm{~mm}$ sensor with 1080p at 120fps capture) in high magnification $(\times 20)$ both in a phaco vitrectomy procedure for a retinal detachment as well as using an artificial training eye. In the latter setting we also used a 1 square metre sheet of white absorbent paper to capture droplet/ aerosol settling using fluorescein dye. The sheet was then examined under magnification $(\times 20)$. The above set up was used to examine the activated instrumentation outside the eye and within the eye, with and without a layer of ophthalmic viscosurgical device (OVD) substance on the ocular surface.

In all settings, a $23 \mathrm{~g}$ vitrectomy probe at $6000 \mathrm{cpm}$ did not show droplet or visible aerosol generation. The 


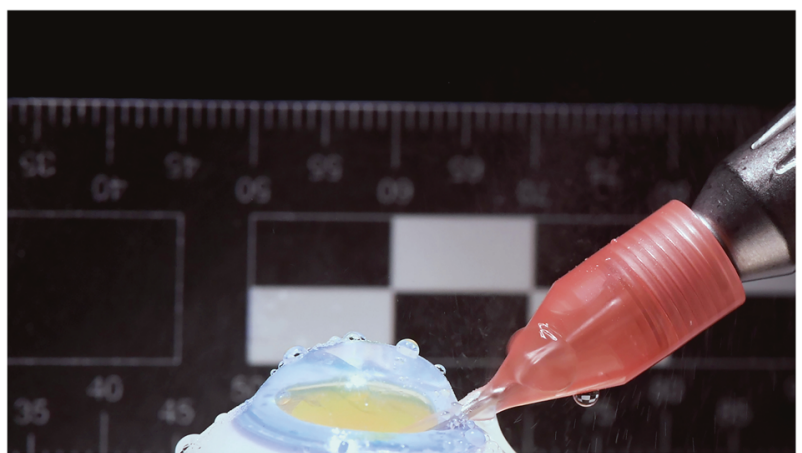

Fig. 1 Visible aerosol generation. A torsional tip demonstrating droplet generation when functioning within a model eye.

phacoemulsification probe functioning in the longitudinal axis showed droplet and aerosol generation with the infusion on, when activated outside the eye. Neither of these phenomena were noted when the phaco was activated within the eye with maximum power setting and burst mode. This was the case in all three settings described above (video link 1). The probe with mixed vibrations along the longitudinal and lateral axes showed visible droplet/aerosol generation, even with the probe within the eye, emanating from the junction between the incision and probe (Fig. 1 and video link 2). The droplets visible under magnification spread up to a radius of $9 \mathrm{~cm}$. This effect was not observed when the cornea and limbus was coated with OVD.

The high speed instrumentation described in other fields, for example dentistry, where the ultrasonic scaler has the closest approximation to the phaco probe, the high speed tip is exposed and the aerosol generation and dispersion follows a funnel shaped, directional pattern [1]. In the phaco probe the sleeve with its column of fluid acts as a damper for the vibration of the shaft with the exposed tip generating droplet and aerosol. However, when placed within the anterior chamber, presumably the fluid within the cavity and the plasticity of the corneal dome acts as a similar damper for the vibrations from the tip. The transmitted vibration from the tip needs to be strong enough at the ocular surface to break the surface tension of the fluid layer to generate droplet/aerosol. The probe with multi axis vibration probably reduces the damping effect of the sleeve near the incision.
The above observations along with recent evidence of low transmission risk for COVID19 from the tear film would justify further study and a reappraisal of existing guidance about phacoemulsification and vitrectomy as potential AGPs. There exist more sensitive techniques for aerosol detection, like laser interferometry, which will be able to provide confirmatory evidence [6].

There still persists the greater risk of transmission from aerosol generation involving the nasopharyngeal mucosa from coughing, sneezing, talking and intubation, which can be mitigated by measures well described elsewhere [7].

Links to videos:

https://www.youtube.com/watch?v=QaJ1sPjjthw

https://www.youtube.com/watch?v=fK255TbzQTA\&fea

ture $=$ youtu.be

\section{Compliance with ethical standards}

Conflict of interest The authors declare that they have no conflict of interest.

Publisher's note Springer Nature remains neutral with regard to jurisdictional claims in published maps and institutional affiliations.

\section{References}

1. Harrel SK, Molinari J. Aerosols and splatter in dentistry: a brief review of the literature and infection control implications. J Am Dental Assoc. 2004;135:429-37.

2. Ivan SYJ, Danielle EA, Adrian EZK, Lin-Fa W, Pooja R, Barnaby $\mathrm{EY}$, et al. Assessing viral shedding and infectivity of tears in coronavirus disease 2019 (COVID-19) patients. Ophthalmology. 2020. https://doi.org/10.1016/j.ophtha.2020.03.026.

3. Packer M, Fishkind WJ, Fine IH, Seibel BS, Hoffman RS. The physics of phaco: a review. J Cataract Refract Surg. 2005;31:424-31.

4. Mietz HF, Wan A, Welsandt. G, Torsional Phacoemulsification A. Technique significantly increases the energy delivered to the anterior chamber during cataract surgery. Invest Ophthalmol Vis Sci 2007;48:1077.

5. Duguid JP. The size and the duration of air-carriage of respiratory droplets and droplet-nuclei. J Hyg (Lond). 1946;44:471-9.

6. Anfinrud P, Stadnytskyi V, Bax CE, Bax A. Visualizing speechgenerated oral fluid droplets with laser light scattering. N Engl J Med. https://doi.org/10.1056/NEJMc2007800.

7. Cook TM. Personal protective equipment during the COVID-19 pandemic - a narrative review. Anaesthesia. 2020. https://doi.org/ 10.1111/anae.15071. 